\title{
Phase-III-Studien zu E25 bei Asthma
}

\section{Symptomatik bessert sich}

Mit dem rekombinanten humanen Anti-IgE-Antikörper rhuMAbE25, kurz E25, steht ein neuartiges Therapieprinzip zur Behandlung von atopischen Erkrankungen wie Asthma oder Rhinitis zur Verfügung. Bei Patienten mit mittelschwerem Asthma kann unter einer regelmäßigen E25-Gabe die Dosis inhalativer Kortikoide gesenkt werden, die Zahl der Asthmaexazerbationen geht zurück - so das Ergebnis einer kürzlich abgeschlossenen Phase-III-Studie.

Deim Asthma bronchiale handelt es Dich um ein multifaktorielles pathogenetisches Geschehen. Allerdings, so W. W. Busse (Madison, Wisconsin/USA), hat jede Zeit ihre „Forschungslieblinge": Während in den 80er Jahren IgE als Auslöser für das allergische Asthma im Mittelpunkt des Interesses stand, können die 90er Jahre als die „Dekade der Lymphozyten, Zytokine, Adhäsionsproteine, Gene und der Entzündung" bezeichnet werden, sagte der für die nächste Amtsperiode designierte Präsident der AAAAI.

\section{Applikation alle 2 bis 4 Wochen}

Seitdem für die Anwendung am Menschen geeignete Anti-IgE-Antikörper wie E25 zur Verfügung stehen, wird nun aber auch dem IgE als einem zentralen Vermittler der Entzündungsreaktion wieder mehr Aufmerksamkeit geschenkt.

Die Resultate klinischer Studien, die mit dem Therapieprinzip der AntiIgE-Antikörpertherapie erzielt werden, sind ermutigend. So stellte Busse die Ergebnisse einer randomisierten, doppelblinden und plazebokontrollierten Phase-III-Studie an 525 erwachsenen Patienten mit einem mittelschweren Asthma vor.

Mit Beginn der über 28 Wochen angelegten Untersuchung erhielten die Asthmapatienten in Abhängigkeit vom Körpergewicht und den Serum-IgESpiegeln den Anti-IgE-Antikörper E25 entweder in einer Dosierung von 150 bis $300 \mathrm{mg}$ alle 4 Wochen oder 225 bis $375 \mathrm{mg}$ alle 2 Wochen oder eine entsprechende Plazebogabe.
Nach einer 16-wöchigen stabilen Therapiephase wurde die Kortikosteroiddosis dann über 12 Wochen alle 14 Tage um 25\% reduziert, um die niedrigst mögliche Dosis für eine optimale Asthmakontrolle zu ermitteln.

Asthmaexazerbationen traten in der Verumgruppe sowohl in der stabilen Therapiephase als auch während der Kortikoid-Reduktionsphase signifikant seltener auf als in der Plazebogruppe.
Busse: „Hinsichtlich der Asthmaexazerbation erwies sich die E25-Therapie als doppelt so protektiv wirksam wie eine Behandlung mit Plazebo."

\section{Kortikoiddosis reduziert}

Auch die Kortikoiddosis konnte in der Verumgruppe um $75 \%$ reduziert werden - von durchschnittlich 430 bis $840 \mu \mathrm{g}$ Beclometason/d zu Beginn der Studie auf $126 \mu \mathrm{g} / \mathrm{d}$ unter zusätzlicher Antikörper-Gabe. E25 verbesserte die Asthmasymptomatik über die gesamte Studienzeit hinweg, der Verbrauch an Bedarfsmedikation während der stabilen Therapiephase sank. Substanzbezogene Nebenwirkungen traten nicht auf.

Die Zulassung für den monoklonalen Antikörper E25 wird voraussichtlich Mitte dieses Jahres beantragt, mit der Marktzulassung ist Mitte $2001 \mathrm{zu}$ rechnen.

\section{$b k$}

\section{Busse W W}

56. Jahrestagung der American Academy of Allergy, Asthma and Immunology (AAAAI), San Diego, 2000.

\section{E25 bei Asthmakindern: Über die Hälfte kann auf Kortikoide ganz verzichten}

Wirksamkeit, Sicherheit und Verträglichkeit von E25 sind auch bei Kindern mit Asthma gut dokumentiert. Eine Phase-III-Studie zeigt: $55 \%$ konnten unter E25 auf inhalative Kortikoide ganz verzichten.

n die Phase-III-Studie waren 334 Kinder ( 6 bis 12 Jahre) mit einem mäßigen bis mittelschweren Asthma einbezogen. Auch hier, so H. Milgrom (Denver, Colorado/USA), ergab sich eine Senkung der Asthmaexazerbationen gegenüber Plazebo. Die E25-Dosierung in dieser 28wöchigen Studie lag bei 150 bis $350 \mathrm{mg}$ alle 4 Wochen oder 250 bis $375 \mathrm{mg}$ alle 2 Wochen - war also der Erwachsenendosierung vergleichbar.

Ergebnis: Die Dosis inhalativer Kortikoide ließ sich bei allen Kindern reduzieren, 55\% der Kinder in der Verumgruppe konnten die inhalativen Steroide sogar ganz absetzen. Der Verbrauch an Bedarfsmedikamenten lag unter der Verumgabe deutlich niedriger als unter Plazebo.
Milgrom: „Kinder profitieren von einer E25-Therapie besonders, da durch die Kortikoideinsparung potenziellen Wachstumseinschränkungen vorgebeugt wird." Möglicherweise wirkt E25 auch präventiv - z.B. hinsichtlich des Fortschreitens des RemodellingProzesses der Atemwege.

Einige Kinder erhalten die AntiIgE-Antikörpertherapie nun bereits über 3 Jahre, erklärte Milgrom. „Die Compliance ist anhaltend hoch und die subkutanen Injektionen alle 2 bzw. 4 Wochen werden sehr gut toleriert." $b k$

\section{Milgrom $\mathbf{H}$}

56. Jahrestagung der American Academy of Allergy, Asthma and Immunology (AAAAI), San Diego, 2000. 